\title{
ON MECHANISMS OF LOW-ENERGY NUCLEAR-CHEMICAL PROCESSES
}

\author{
${ }^{1,2}$ Serge Timashev \\ ${ }^{1}$ Karpov Institute of Physical Chemistry, http:/ /www.karpovipc.ru \\ Moscow 105064, Russian Federation \\ ${ }^{2}$ National Research Nuclear University MEPhI, https://mephi.ru \\ Moscow 115409, Russian Federation \\ serget@mail.ru
}

Abstract. It is shown that a wide variety of low-energy nuclear transformations studied under conditions of a nonequilibrium low-temperature glow discharge plasma and laser ablation of metals in aqueous media can be understood on the basis of the concepts of the dynamic interrelation between the electron and nuclear subsystems of an atom. The initiating role in such processes belongs to electrons a sufficiently large kinetic energy $\mathrm{E}_{\mathrm{e}} \sim 3-5 \mathrm{eV}$ (by chemical scales), which they can acquire under the indicated conditions. Inelastic scattering of electrons by nuclei in according to weak nuclear interaction becomes possible in the collision of such electrons with ions or plasma atoms (here we assume that the nuclei are not related to "K-capture" nuclei). At the first stage of such a nuclear-chemical interaction, a nucleus arise, the charge of which is one unit less than the charge of the initial nucleus, and nuclear matter is locally disrupted: the nuclear mass in this case is insufficient to preserve nuclear matter in the base state of interacting nucleons. Under such anomalous excitations of nuclear matter, which are characterized as the states of "inner shake-up" or isu-state, the relaxation dynamics of the nuclei is initiated by weak nuclear interactions. Such nuclei, being $\beta$-active (“ $\beta$-nuclei”), can have sufficiently long lifetimes and effectively participate in nuclear reactions (as the $\beta$-neutron and $\beta$-dineutron introduced into consideration). If the initial nucleus is radioactive, the decay of " $\beta$-nuclei" will occur with a probability many orders of magnitude greater than the decay probability of the original nuclei. As an example, the nuclearchemical transformations realized in the E-CAT reactor of Andrea Rossi are also considered.

Key words: low-temperature plasma, laser ablation of metals in aqueous media, $\beta$-nuclei, low-energy nuclear-chemical transformation, $u$ - and d-quarks, Feynman diagrams of the initiating radioactive decay.

PACS 25.10.+s

Bibliography -43 references

Received 25.5.2017

RENSIT, 2017, 9(1):37-51

DOI: $10.17725 /$ rensit.2017.09.037

CONTENTS

1. INTRODUCTION (37)

2. Electronic FaCtor IN INITIATING NUCLEAR PROCESSES (39)

3. Possible mechanisms of NuClear-CHEmical REACTIONS (42)

3.1. Mechanism of NUClear FUSion (43)

3.2. Mechanism of $\mathrm{e}^{-}$-catalysis (44)

3.3. Harpoon mechanism (45)

4. Nuclear chemical processes in ANDrea Rossi's E-CAT REACTOR (46)

5. Concluding Remarks(48)

REFERENCES (49)

\section{INTRODUCTION}

Beginning with the work of Fleischmann, Pons and Hawkins [1] on observation of excessive heat generation during the electrolysis of $\mathrm{D}_{2} \mathrm{O}$ heavy water with a Pd cathode, when the generation of neutrons and tritium was recorded, it became clear that the problem of establishing the mechanism of the occurring nuclear transformations could become a key to solving the complex of problems that arose in connection with the phenomenon of low-energy nuclear reactions (LENR). One of the first attempts to solve this problem was associated with the possibility of the existence of a stable dineutron with a binding energy $\varepsilon_{\mathrm{dn}}$ less than 
$3.01 \mathrm{eV}$ (for the deuteron not to be radioactive) [2]. In the experiment [1], the arising a dineutron as a result of the interaction of an activated electron with a deuteron under conditions of electrolysis of heavy water could be considered as the first stage preceding the reaction of formation of tritium during the interaction of the dineutron with the deuteron. However, there remained questions. It was unclear whether it is possible to actually activate the electrons in the conditions of electrolysis of heavy water on a palladium cathode to the necessary (what exactly?) energies, and whether there really is a dineutron. The possibility of the ${ }^{2} n$ nucleus existence was discussed back in the early 1960s when studying the $T\left(d,{ }^{2} n\right)^{3} \mathrm{He}$ process using the ${ }^{27} A l\left({ }^{2} n, \gamma\right)^{29} A l$ reaction for the detection of ${ }^{2} n$ particles [3]. However, in [4], which appeared shortly after the publication of [3], the results of a similar experiment were reported and it was reported that the yield of ${ }^{29} \mathrm{Al}$, confirming the reality of the existence of ${ }^{2} n$ nucleus, was observed only at the background level.

Moreover, it was known, based on general considerations, that the ${ }^{2} n$ nucleus can not exist in principle. Since there is a bound state in the neutron-proton system with a binding energy $\varepsilon_{\mathrm{D}}=2.22 \mathrm{MeV}$ and $\operatorname{spin} S=1$, and also there is a virtual level with energy $70 \mathrm{keV}$ and spin $S=0$, for the system of two neutrons only the existence of a virtual state with spin $S=0$ due to the charge independence of the nuclear forces (isotopic invariance), is possible [5]. But in this state there should be a weak repulsion, and the state with $S=1$ can not exist due to the Pauli principle. Nevertheless, in this review [5], based on an analysis of the experimental data available at that time (1965), an upper estimate of the cross section for the formation of a nuclear stable dineutron, $\mathrm{s}<0.001-0.01 \mathrm{mb}$, was still given, although this estimate practically excluded the possibility of manifestation of ${ }^{2} n$ in any experiments. This estimate was confirmed by the result of a much later experiment [6], in which for the cross section of occurring a stable dineutron in the interaction of cold neutrons with deuterons in the reaction $n+d \rightarrow{ }^{2} n+p$, we obtained: $\sigma \leq 1$ mcb.
With time, it became clear that in order to understand the results of [1], as well as many later works on initiating low-energy nuclear reactions (LENR) and accelerating radioactive $\alpha$ and $\beta$ decays, including under low-temperature plasma conditions [7-9], and when laser ablation of metals in aqueous media [10-13], the idea of a stable dineutron should be abandoned, and it is necessary to involve other hypotheses. At the same time, the results of [14] in which the spectrum of the "lost mass" in the process ${ }^{6} \mathrm{Li}\left(\pi^{-}, p\right)^{5} \mathrm{H}$ (see Fig. 1) with the kinetic energy of $\pi^{-}$-mesons equal to $125 \mathrm{MeV}$ was investigated, continue to cause surprise. It follows from Fig. 1, a fixed maximum in the region of the lost mass from zero to $-5 \mathrm{MeV} / \mathrm{c}^{2}$ could well correspond to a hypothetical dineutron with a binding energy of $\varepsilon_{\mathrm{dn}} \approx 3 \mathrm{MeV}$. However, in this case, the probability of the occurring a dineutron in the reaction involving a $\pi^{-}$-meson would have an order of

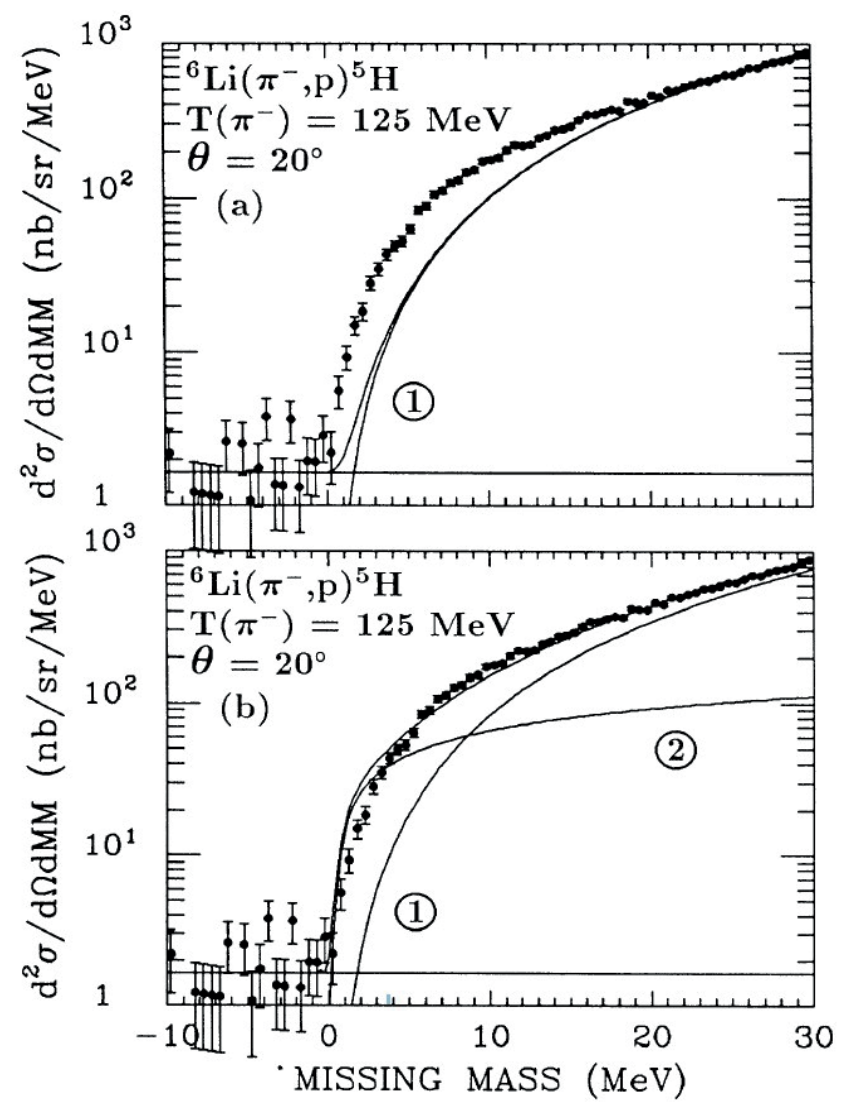

Fig. 1. The missing-mass spectra for the reaction ${ }^{6} \mathrm{Li}\left(\pi^{-}, p\right)^{5} \mathrm{H}$ [14]. The constant line at the bottom corresponds to the background. The phase-space distributions shown are as follows: (a) curve 1, fit for ${ }^{5} \mathrm{H} \rightarrow{ }^{3} \mathrm{H}+n+n$; (b) best-fit curve as sum of curve 1 for ${ }^{5} \mathrm{H}$ $\rightarrow{ }^{3} \mathrm{H}+n+n$ and curve 2 for ${ }^{5} \mathrm{H} \rightarrow{ }^{3} \mathrm{H}+{ }^{2} n$. 
magnitude higher than the values indicated above, for unknown reasons.

In subsequent years, in order to understand the results of many papers in which low-energy nuclear transformations and low-temperature plasma accelerated decays of radioactive nuclei were investigated, attempts were repeatedly made to introduce neutral particles with a baryon number of two (or one) that were the weakly coupled or resonant state of the deuteron (or proton) with an electron or a neutron with a neutrino. Such particles could participate in low-energy nuclear reactions, since for such a type of particles there would be no problem of overcoming the "Coulomb barrier" in nuclear interactions. We do not consider such possibilities here, since in accordance with the concepts of nuclear physics, electron and neutrino localization on nuclear scales $\sim 10^{-13} \mathrm{~cm}$ would have an abnormally high, physically incredible uncertainty in the pulses [15].

In this paper, it is shown that the problems of establishing the possible mechanisms of LENR processes discussed above can be understood on the basis of the concepts of the interrelation between the electronic and nuclear subsystems of an atom [16-20], which determines the possibility of initiating the entire variety of nuclear transformations, including those studied in mentioned works [7-13], as well as realized in the Andrea Rossi E-CAT reactor [21, 22].

\section{ELECTRONIC FACTOR IN}

\section{INITIATING NUCLEAR PROCESSES}

According to the phenomenological understanding [17-20], the dynamic interrelation between the electron and nuclear subsystems of atom, realized through the electromagnetic component of the physical vacuum, the EM vacuum, is the key factor in initiating low-energy nuclear reactions [7-13] and the processes of radioactive decay of nuclei $[13,17,18]$. A reflection of this interrelation is, in particular, the experimentally established facts showing that the possibility of radioactive decay of nuclei is determined by the positive difference between the mass of the initial nucleus together with the mass of the electron subsystem of the atom (i.e., the mass of the whole atom, not the nucleus) and the total mass of the decay products $[23,24]$. Usually, when considering the mechanisms of these low-energy nuclear processes and the decay of atomic nucleus ${ }_{Z}^{A} N$ ( $\mathrm{Z}$ and $\mathrm{A}$ are the atomic number and mass number of the nucleus $N$, respectively), nuclear matter is represented in the form of interacting nucleons. For example, in the $K$-capture, when the electron of the inner shells of the atom interacts with the surface of the nucleus and a new daughter nucleus is formed, the nucleon structure of nuclear matter does not change. At the initial, irreversible stage of this process, the electron emits a neutrino $v$ when interacting with the nucleus surface. The formed virtual vector $W^{-}$-boson, which is introduced into the nuclear matter, is transformed into a $d$-quark when interacting with the $u$-quark of one of the protons, as a result of which this proton turns into a neutron, and the nucleus ${ }_{Z-1}^{A} M$ is formed. However, the situation can drastically change when the $K$-capture is energetically forbidden (it is such cases that are considered below), but the electron has a sufficiently large (by chemical standards) kinetic energy $E_{\mathrm{e}} \sim 3-5 \mathrm{eV}$, as it can be realized in low-temperature plasma. Under these conditions, when the process of ionization of electron shells by such electrons is not yet realized, then, during scattering of electrons with the indicated kinetic energy and the corresponding de Broglie wavelength $\lambda \approx 0.5 \mathrm{~nm}$, the oscillation dynamics of the electron subsystems of atoms and ions is initiated on atoms and ions and, thus, the probability of interaction between the electrons of the inner subshells of atoms and ions and the corresponding nuclei increases.

At the first, irreversible stage of such interaction, a neutrino $v$ is emitted and a vector $W^{-}$-boson is introduced into the nuclear matter of the original nucleus ${ }_{Z}^{A} N$ according to the relation: ${ }_{Z}^{A} N+e_{b e}^{-} \rightarrow{ }_{Z-1}^{A} M_{i s u}+v$.

As a consequence, the nucleon structure of the formed nuclei ${ }_{Z-1}^{A} M_{i s u}$, whose charge is less than the charge of the initial nucleus by one elementary charge, turns out to be locally disrupted. Indeed, the vector $W^{-}$-boson, when interacting with the 
$u$-quark of one of the protons of the ${ }_{Z}^{A} N$ nucleus, can only yield the formation of a virtual $d$-quark with a subsequent chain of virtual transformations of quarks involving vector $W$-bosons, but a neutron cannot be formed due to the deficit of the total mass of such a nucleus. The emerging state of local anomaly of nuclear matter with a broken nucleon structure is characterized as a metastable state of "inner shake-up" or isu-state. The latter is indicated by the subscript in the notation of the nucleus in the right-hand side of the relation (1). The lower index in the notation for the electron in the left-hand side of (1) indicates the activated nature of this stage of the process. The initiated chain of virtual transformations of quarks with the participation of the vector $W$-bosons should be interrupted in the irreversible decay of the virtual $W^{-}$-boson with the formation of the initial nucleus, an electron, and an antineutrino $\tilde{v}$ :

$$
{ }_{Z-1}^{A} M_{i s u} \rightarrow{ }_{Z}^{A} N+e^{-}+\tilde{v},
$$

so that the gross process can be represented in the form of inelastic scattering of an electron on the initial nucleus:

$$
{ }_{Z}^{A} N+e_{b e}^{-} \rightarrow{ }_{Z}^{A} N+e^{-}+v+\tilde{v} .
$$

The nuclei with the state of nuclear matter in the metastable isu-state of the "internal shake" will be called the " $\beta$-nuclei". The threshold energy of such a process with the production of a $v \tilde{v}$ pair, determined by the rest masses of neutrinoantineutrinos, is about $0.3 \mathrm{eV}$ [25].

As is known, the nucleus is a system of nucleons connected in a single whole by means of exchange interactions by exchanging quarks via pions. Therefore, the formation in the nucleus of three quarks unconnected into a nucleon, which can then be regarded as "markers" of new degrees of freedom, in fact, means that the intensity of nuclear forces is insufficient to provide the traditional, proton-neutron organization of nuclear matter in the system under consideration. The subsequent relaxation dynamics of the locally appeared isu-state, which can be transmitted to other nucleons of the nucleus by means of pions, is initiated only by weak nuclear interactions, which are realized through quarks during creation and absorption of the gauge vector neutral
$Z^{0}$ - and the charged $W^{ \pm}$-bosons. In the case under consideration, such a relaxation terminates with the decay of the virtual vector $W^{-}$-boson with the formation of the initial nucleus during the emission of an electron and antineutrino. The lifetime of the formed $\beta$-nuclei in the metastable isu-state can be rather considerable, from tens of minutes to several years, and the nuclei in this state can directly participate in a variety of nuclear processes [17, 18].

It must also be taken into account here that the relaxation reorganization of nuclear matter in the process of formation of products of such nuclear transformations is carried out, first of all, by the formation of a purely nucleon structure of the nucleus, in accordance with the principle of least action. While in the nuclei with the proton-neutron, nucleon structure, the relaxation processes of de-excitation can go through the excited states of the nucleus and include the stages of emission of $\gamma$-quanta, in the $\beta$-nuclei such relaxation is practically impossible. Therefore, if the atomic nuclei with a partial "non-nucleon" state of nuclear matter participate in the processes, then the mechanism of relaxation of the formed products is inevitably associated with energy loss by the emission of neutrino-antineutrino pairs, or the URCA process [26], and not by the emission of $\gamma$-quanta by excited nuclei, as is the case during the relaxation of nuclear products having the proton-neutron organization of nuclear matter. It is for this reason that the corresponding nuclear processes are safe for the environment.

Of special interest are the cases when the formation of isu-states in nuclear matter is initiated in the initially radioactive nuclei, since the relaxation process with the decay of the vector $W^{-}$-boson can initiate a general radioactive decay of the nucleus in the isu-state with the creation of the daughter products of the decay of the initial radioactive nucleus. According to $[18,27]$, the violation of the general stability of nuclear matter in the metastable isu-state occurs as a result of a change in the boundary conditions for the components of the electric field intensity vector of the EM vacuum on the surface of the nucleus in whose volume such a violation of the 
nucleon structure has occurred. An indicator of the instability of the ${ }_{Z-1}^{A} M_{i s u}$ nucleus arising during process (1) is the absolute value of the structural energy deficit $\Delta Q(\Delta Q<0)$ of this nucleus in the metastable isu-state, defined as $\Delta Q=\left(m_{A_{Z} N}-m_{Z \rightarrow-1}^{A}\right) c^{2}$. In this case, the mass of the

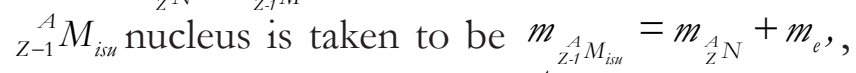
where $m_{Z}^{A}$ is the mass of the ${ }_{Z}^{A} N$ nucleus and $m_{\mathrm{e}}$ is the rest mass of the electron.

For example, during the laser ablation of metal samples in the aqueous solution of uranyl, when low-temperature plasma forms in the vapor medium adjacent to the metal surface, the initiating interaction of plasma electrons with the ${ }^{238} U$ nuclei leads to the formation of the " $\beta$-protoactinium" nuclei and subsequent $\beta$-decay of the ${ }_{91}^{238} \mathrm{~Pa}_{i s u}$ nuclei with the formation of thorium-234 and helium-4 nuclei as the products of decay of the initial uranium-238 nucleus:

$$
\begin{aligned}
& { }_{92}^{238} \mathrm{U}+e_{b e} \rightarrow{ }_{91}^{238} \mathrm{~Pa}_{i s u}+v \rightarrow \\
& \rightarrow{ }_{90}^{234} \mathrm{Th}+{ }_{2}^{4} \mathrm{He}+e^{-}+v+\tilde{v}+Q(4.27 \mathrm{MeV}) .
\end{aligned}
$$

In this case, the effective rate constant of such initiated decays of ${ }^{238} \mathrm{U}$ nucleus, as shown in [17], increases by 9 orders of magnitude, so that a kind of " $\mathrm{e}^{-}$-catalysis" is realized. The deficit $\Delta Q$ of the structural energy of the formed nucleus of $\beta$-protoactinium is $\Delta Q \approx-3.46 \mathrm{MeV}$. An unexpected result was obtained while carrying out experiments with a beryllium sample. It turned out that the beryllium nanoparticles, formed in the solution after the completion of the laser action for an hour, exhibited an anomalously high rate of formation of thorium-234 nuclei for more than 500 days after completion of laser ablation. The half-life period for the nuclei, initiated under laser ablation and producing thorium-234, was 2.5 years. This phenomenon could naturally be associated with the accumulation of $\beta$-protoactinium nuclei in beryllium nanoparticles during laser ablation, which lasted only for an hour.

We also give examples $[17,20]$ of $\beta^{-}$-decay of ${ }_{27}^{60} \mathrm{Co},{ }_{55}^{137} \mathrm{Cs}$ and ${ }_{56}^{140} \mathrm{Ba}$ nuclei, initiated by the $\mathrm{e}^{-}$catalysis mechanism, the half-life $T_{1 / 2}$ of which is 1925 days, 30.1 years and 12.8 days, respectively:

$$
\begin{aligned}
& { }_{27}^{60} \mathrm{Co}+e_{b e}^{-} \rightarrow{ }_{26}^{60} \mathrm{Fe}_{i s u}+v \rightarrow \\
& \rightarrow{ }_{28}^{60} \mathrm{~N}+2 e^{-}+v+2 \tilde{v}+Q(2.82 \mathrm{MeV}), \\
& { }_{55}^{137} \mathrm{Cs}+e_{b e}^{-} \rightarrow{ }_{54}^{137} \mathrm{Xe}_{i s u}+v \rightarrow \\
& \rightarrow{ }_{56}^{137} \mathrm{Ba}+2 e^{-}+v+2 \tilde{v}+Q(1.18 \mathrm{MeV}), \\
& { }_{56}^{140} \mathrm{Ba}+e_{b e}^{-} \rightarrow{ }_{55}^{140} \mathrm{Cs}_{i s u}+v \rightarrow \\
& \rightarrow{ }_{57}^{140} \mathrm{La}+e^{-}+v+2 \tilde{v}+Q(1.05 \mathrm{MeV}) .
\end{aligned}
$$

In these cases, the deficit of the structural energy $\Delta Q$, which is lacked by ${ }_{26}^{60} \mathrm{Fe}_{i s u},{ }_{54}^{137} \mathrm{X} e_{\text {iss }}$ and ${ }_{55}^{140} \mathrm{Cs}$ isu nuclei in the "in-shake-up" state to form the basic state of nuclear matter characteristic to ${ }_{26}^{60} \mathrm{Fe},{ }_{54}^{137} \mathrm{Xe}$ and ${ }_{55}^{140} \mathrm{Cs}$ nuclei, is $-0.237 \mathrm{MeV},-4.17 \mathrm{MeV}$ and $-6.22 \mathrm{MeV}$, respectively. It can be expected that the initiating influence of electrons on the $\beta^{-}$decay of nuclei in low-temperature plasma will be manifested to the greatest extent in the cases when the "mismatch" in the absolute magnitude of the deficit $\Delta Q$ of the structural energy for the forming nuclei in the $i s u$-state is the greatest. Therefore, in the cases under consideration, the effect of accelerating radioactive decay should have been manifested for ${ }_{55}^{137} \mathrm{Cs}$ and ${ }_{56}^{140} \mathrm{Ba}$ nuclei, while for ${ }_{27}^{60} \mathrm{Co}$ nuclei, it should have been minimal. The available experimental data [28] on the initiated decays of ${ }_{55}^{137} \mathrm{Cs},{ }_{56}^{140} \mathrm{Ba}$ and ${ }_{27}^{60} \mathrm{Co}$ agree with this conclusion: the half-life of $\beta^{-}$active cesium-137 and barium-140 nuclei, equal to 30.1 years and 12.8 days, respectively, decreased to about 380 days and 2.7 days, while the halflife of cobalt-60, equal to 1925 days, remained practically unchanged. The Feynman diagrams corresponding to the $\beta^{-}$-decay processes, as well as the positron $\beta^{+}$-decays of nuclei initiated by the $\mathrm{e}^{-}$-catalysis mechanism, are shown in Fig. 1.

The unexpectedness of the presented result about the possibility of external influences on the dynamics of the decay of a radioactive nucleus is that this effect is associated with electrons that cannot interact with nucleons of the nucleus as fragments of nuclear matter, but can initiate (through the vector $W^{-}$-bosons) local disturbances in the nucleon structure of the nucleus. At the same time, as experience shows, the external excitation of the radioactive nucleus as an integral system (in particular, under the influence of 
$\gamma$-radiation) cannot affect the radioactive decay rate, and therefore, the discussed phenomenon of initiating the instability of the nucleus. In these cases, nuclear matter manifests itself as an integral system of interacting nucleons with their inherent individual characteristics.

The Feynman diagrams for the $\beta^{-}$and $\beta^{+}$decays as well as for the $\alpha$-decay, initiated by the $\mathrm{e}^{-}$-catalysis mechanism, are shown in Fig. 2 a-d. In the analysis of the processes presented in Fig. 2, we must have in mind that weak nuclear interactions are not as weak as it is often assumed: the value of the corresponding dimensionless constant $\alpha_{F}$ is almost an order of magnitude greater than the value $\alpha_{\mathrm{e}}$ of the fine structure constant $[15,27]$. Indeed, if we take $\alpha_{\mathrm{s}}=\sqrt{2}$ as the dimensionless constant of strong nuclear interaction [27], then taking into account the value of the square of the "elementary charge of weak nuclear interaction" $q_{F}^{2} \equiv G_{F} / a_{Z}^{2} \quad$ [29], where $a_{\mathrm{z}}=2^{1 / 2} \mathrm{~h} / m_{\mathrm{z}} c \approx 3.310^{-16} \mathrm{~cm}$ is the characteristic radius associated with the mass of the intermediate $Z^{0}$ vector boson $\left(m_{\mathrm{Z}}=91.2\right.$

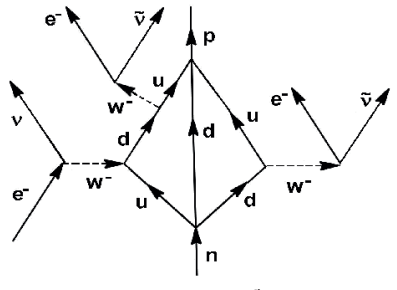

$a$
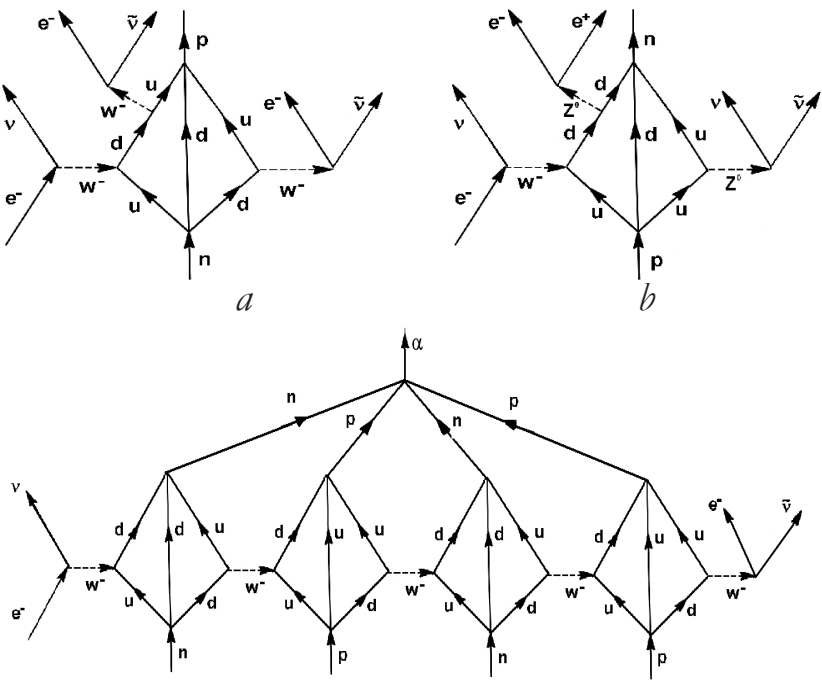

$c$

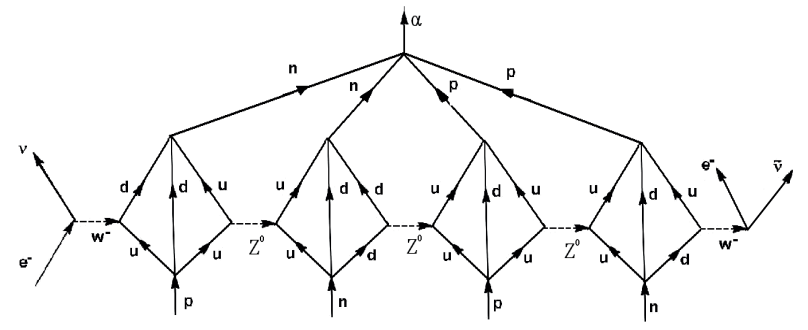

d

Fig. 2. Feynman diagrams for the initiating $\beta^{-}$-decay (a), $\beta^{+}$-decay (b) and the a-decay $(c, d)$.
$\mathrm{GeV} / \mathrm{c}^{2}=1.62 \cdot 10^{-22} \mathrm{~g}$ ), and $G_{\mathrm{F}}=1.17 \cdot 10^{-5}(\mathrm{hc})^{3} /$ $(\mathrm{GeV})^{2}$ is the Fermi constant of the four-fermion interaction, we obtain for the quantity $\alpha_{F}: \alpha_{F}=\frac{q_{F}^{2}}{\hbar_{c}}$ $\approx 4.9 \cdot 10^{-2}$, so that $\alpha_{\mathrm{F}} / \alpha_{\mathrm{s}} \approx 3.45 \cdot 10^{-2}$. Remind that $\alpha_{\mathrm{e}}=1 / 137 \approx 0.73 \cdot 10^{-2}$, so that $\alpha_{\mathrm{e}} / \alpha_{\mathrm{s}} \approx 5.2 \cdot 10^{-3}$ and $\alpha_{\mathrm{F}} / \alpha_{\mathrm{e}}=6.7$. Unfortunately, very often in the literature, in the estimation of the dimensionless constant of weak nuclear interaction, the proton mass is used as the normalization mass which is almost 100 times smaller than the mass of the $\mathrm{Z}^{0}$ vector boson. For this reason, the value of the constant $\alpha_{F}$ is underestimated by almost 4 orders of magnitude. The real value of this constant, in accordance with the above estimates, is only 35 times, not 5 orders of magnitude less than the dimensionless constant of the strong nuclear interaction.

In conclusion of this section, let us pay attention to some unexpectedness of the presented result about the possibility of external influences of electrons on the dynamics of the decay of a radioactive nucleus. It turns out that although the electrons can not interact with the nucleons of the nucleus as fragments of nuclear matter, they can initiate (through vector $\mathrm{W}^{-}$-bosons) local disturbances in the nucleon structure of the nucleus At the same time, as experience shows, the external excitation of the radioactive nucleus as an integral system (under the influence of $\gamma$-radiation, in particular) can not affect the rate of radioactive decay, and therefore, of the discussed phenomenon of initiating of the metastable state of the nucleus. In these cases, nuclear matter manifests itself as an integral system of interacting nucleons with inherent individual characteristics.

\section{POSSIBLE MECHANISMS OF NUCLEAR-CHEMICAL REACTIONS}

The simplest $\beta$-nuclei are the $\beta$-neutron and $\beta$-dineutron, which can be formed by the interaction of high-energy electrons with protons or deuterons, for example, by laser ablation of metals in ordinary or heavy water, and also under conditions of protius-containing or deuteroncontaining glow discharge plasma, respectively, according to 


$$
\begin{aligned}
& p^{+}+e_{b e}^{-} \rightarrow{ }^{1} n_{i s u}+v, \\
& d^{+}+e_{b e}^{-} \rightarrow{ }^{2} n_{i s u}+v .
\end{aligned}
$$

If the half-lives $T_{1 / 2}$ of such $\beta$-nuclei are sufficiently long, the neutral nuclei ${ }^{1} n_{\text {isu }}$ and ${ }^{2} n_{\text {isu }}$, respectively, with baryon numbers equal to one and two, zero lepton charges and rest masses equal to the masses of the hydrogen atom and deuterium, can effectively participate in a variety of nuclear processes [13, 17-20].

It was shown earlier that the half-life $T_{1 / 2}$ of the $\beta$-dineutron:

${ }^{2} n_{i s u} \rightarrow d^{+}+e^{-}+\tilde{v}$

with the formation of a deuteron, an electron, and an antineutrino, turns out to be sufficiently long, at least tens of minutes. This conclusion is based on an analysis of the experimental data on the synthesis of tritium nuclei $\mathrm{t}^{+}$upon the laser ablation of metals in heavy water [13]. It was assumed that the latter process occurs upon interaction between tritium nucleus $\mathrm{t}^{+}$and $\mathrm{a}^{2} n_{\text {isu }}$ nucleus:

$$
d^{+}+{ }^{2} n_{\text {isu }} \rightarrow \mathrm{t}^{+}+n+Q(3.25 \mathrm{MeV})
$$

where $n$ is a neutron. At the same time, another process can take place alongside (12):

$$
d^{+}+{ }^{2} n_{i s u} \rightarrow{ }_{2}^{3} \mathrm{He}+n+e^{-}+\tilde{v}+\mathcal{Q}(3.27 \mathrm{MeV}),
$$

as a result of weak nuclear interaction.

In [13], the possibility of formation of a hypothetical $\beta$-trineutron ${ }^{3} n_{\text {isu }}$ under the interaction of electrons with tritium nuclei $\mathrm{t}^{+}$was also postulated:

$$
t^{+}+e_{b e}^{-} \rightarrow{ }^{3} n_{i s u}+v
$$

The rest mass of the introduced ${ }^{3} n_{\text {isu }}$ neutral nucleus was assumed to be equal to the rest mass of the tritium atom. It is through the formation of the nucleus ${ }^{3} n_{\text {isu }}$ that the process of initiation of decay of tritium nuclei under conditions of laser ablation of metals in aqueous media found in [13], along with the process of synthesis of tritium nuclei, can occur:

$$
\begin{aligned}
& t^{+}+e_{b e}^{-} \rightarrow{ }^{3} n_{i s u}+v \rightarrow \\
& \rightarrow{ }_{2}^{3} \mathrm{He}+2 e^{-}+v+2 \tilde{v}+Q(0.019 \mathrm{MeV}) .
\end{aligned}
$$

It should be noted here that according to [13], halflife $T_{1 / 2}$ of the decay of the ${ }^{3} n_{\text {isu }}$ nucleus during $\mathrm{e}^{-}$-catalysis turns out to be of the same order of magnitude as the half-life of the ${ }^{2} n_{\text {isu }}$ nucleus, which is many orders of magnitude shorter than that of the tritium nucleus ( $T_{1 / 2}=12.3$ years).

As was shown in [18], the introduction of representations of sufficiently long-lived $\beta$-nuclei formed in a glow discharge in a deuteriumcontaining gaseous medium made it possible to understand the totality of the data $[8,9]$ for initiating the radioactive decay of $\mathrm{W}$ nuclei in the near-surface layers of a tungsten cathode (foil). Here it should be pointed out that although 5 isotopes of tungsten $\left({ }_{74}^{180} \mathrm{~W},{ }_{74}^{182} \mathrm{~W},{ }_{74}^{183} \mathrm{~W},{ }_{74}^{184} \mathrm{~W}\right.$ and $\left.{ }_{74}^{186} \mathrm{~W}\right)$ are potentially $\alpha$-radioactive nuclei:

${ }_{74}^{74} \mathrm{~W} \rightarrow{ }_{72}^{A-4} \mathrm{Hf}+{ }_{2}^{4} \mathrm{He}+Q_{A}$,

they are usually considered as stable isotopes because of the anomalously large period of their half-life, $T_{1 / 2}=10^{17}-10^{19}$ years, which is many orders of magnitude greater than the existence time of Universe. The heat release value $Q_{\mathrm{A}}$ upon the radioactive $\alpha$-decay of tungsten nuclei with mass numbers A, equal to $180,182,183,184$ and 186, is $2.52,1.77,1.68,1.66$ and $1.12 \mathrm{MeV}$, respectively. Based entirely on energy consideration, it was possible to anticipate $\alpha$-decays with the formation of several $\alpha$ particles for the indicated stable isotopes of tungsten, including decay with the formation of nine $\alpha$ particles for the tungsten-180 isotope.

Within the framework of the developed ideas on the formation of nuclei in an unstable isu state, three mechanisms can be distinguished for initiating nuclear transformations, including radioactive decays of nuclei.

\subsection{Mechanism of NUClEAR FUSION}

The fairly long-lived neutral particles $\left({ }^{\mathrm{A}} n_{\text {isu }}, A=\right.$ $1,2,3)$ formed in the low-temperature plasma can diffuse along grain boundaries deep into a cathode and interact with the metal (tungsten) nuclei in its near-surface layers. Excited ${ }_{74}^{A+2} W^{*}$ nuclei can thus appear at the initial stage upon interaction between ${ }^{2} n_{\text {isu }}$ and ${ }_{74}^{A} W$ isotopes and their fusion. In addition to the overall excitation energy (shows an asterisk) about of $10 \mathrm{MeV}$ with respect to the main state of these nuclei, the nuclear matter of such nuclei may be partially (due to the fusion with ${ }^{2} n_{\text {isu }}$ ) in an unbalanced isu-state with loss of stability in the nuclear bulk; 
this leads to subsequent transformation with the emission of $\alpha$ particles and daughter isotopes. It should also be emphasized here that, in contrast to the nuclear reactions that occur upon the collision of reagents in the gaseous phase, the energy factor alone is sufficient for the considered nuclear transformations in the area of grain boundaries of a solid metal phase, due to the possible effects of the medium (without spin and parity matching conditions for the colliding and final nuclei).

When studying the processes occurring under conditions of a glow discharge in a deuteriumcontaining gas medium, the formation of new elements in the near-surface layer of a tungsten cathode after plasma treatment lasting 4 to 7 hours $[8,9]$, the formation not only the stable isotopes of erbium, ytterbium, lutetium and Hafnium, but also radioactive isotopes of ytterbium and hafnium, was detected. If the appearance of stable isotopes in this case could still be hypothetically associated with the diffusion of impurity elements from the cathode volume to the cathode surface processed in the plasma, the formation of radioactive isotopes unambiguously indicates the radioactive decay of tungsten isotopes. Since all the possible reactions of the initiated decay of different isotopes of tungsten are written out in [18], here we give for illustration a few examples:

$$
\begin{aligned}
& { }_{74}^{180} \mathrm{~W}+{ }^{2} n_{\text {isu }} \rightarrow \\
& \rightarrow{ }_{70}^{169} \mathrm{Yb}+{ }_{4}^{9} \mathrm{Be}+{ }_{2}^{4} \mathrm{He}+2 e+2 \tilde{v}+Q(10.09 \mathrm{MeV}),(1 \\
& { }_{74}^{182} \mathrm{~W}+{ }^{2} n_{i s u} \rightarrow \\
& \rightarrow{ }_{70}^{171 m} \mathrm{Yb}+{ }_{4}^{9} \mathrm{Be}+{ }_{2}^{4} \mathrm{He}+2 e-2 \tilde{v}+Q(10.34 \mathrm{MeV}),(17) \\
& { }_{74}^{182} \mathrm{~W}+{ }^{2} n_{i s u} \rightarrow{ }_{72}^{180 m} \mathrm{Hf}+{ }_{2}^{4} \mathrm{He}+\mathcal{Q}(10.26 \mathrm{MeV}), \\
& { }_{74}^{184} \mathrm{~W}+{ }^{2} n_{i s u} \rightarrow{ }_{70}^{178} \mathrm{Yb}+2{ }_{2}^{4} \mathrm{He}+\mathcal{Q}(12.28 \mathrm{MeV}) .
\end{aligned}
$$

Under considering the processes (16)-(19), it was taken into account that in the mass spectra of products, in addition to the major peaks with masses ranging from 169 to 180, the appearance and then the subsequent increase in the peak with mass 9 was recorded. We should also explain that the missing product with a basic mass of 4 corresponding to helium nuclei in the mass spectra obtained in $[8,9]$ was due to the extremely low solubility of helium in tungsten [30] and its high diffusion coefficient in the area of the foil's intergranular boundaries. It is obvious that in order for such processes to proceed, the lifetime of the ${ }^{2} n_{\text {isu }}$ must be long enough for the diffusive permeation of these neutral nuclei into the nearsurface areas of the foil along the grain boundaries. This agrees with the conclusion in [13] that the time must be no less than tens of minutes when synthesizing tritium under conditions of the laser ablation of metals in heavy water.

Obviously, the $\beta$-dineutron plays the same role in the realization of low-energy nuclear reactions, which previously was hypothetically assigned to a stable dineutron. And now the conclusions about the smallness of the possible contribution of the dineutron to the probability of the process $n+d \rightarrow$ ${ }^{2} n+p[5,6]$ become clear, as well as the noticeable probability of the formation of the dineutron in the reaction ${ }^{6} \mathrm{Li}\left(\pi^{-}, p\right)^{5} \mathrm{H}$ initiated by the meson [14]. Indeed, in the interaction of the $\pi^{-}$-meson as a quark-antiquark pair with a nucleus, the quark structure of nucleons should manifest itself, and the possibility of forming a long-lived nucleus ${ }^{2} n_{\text {isu }}$ opens up. Therefore, most likely, the maximum in the dependence shown in Fig. 1 in the region of the "missing mass" is determined precisely by the formation of a nucleus ${ }^{2} n_{\text {isu }}$ whose mass is equal to the mass of the deuterium atom. The "missing mass" $\Delta$ (in $\mathrm{MeV}$ units) in this case, in accordance with the maximum position in Fig. 1, is $\Delta=\left(m_{\mathrm{n}}-m_{\mathrm{p}}-m_{\mathrm{e}}\right) c^{2}-\varepsilon_{\mathrm{d}} \approx 3.01 \mathrm{MeV}$, where $m_{\mathrm{n}}$, $m_{\mathrm{p}}$ and $m_{\mathrm{e}}$ are the masses of the neutron, proton and electron, respectively, and $\varepsilon_{\mathrm{d}}$ is the deuteron binding energy. As for the nuclear reaction $n+$ $d \rightarrow{ }^{2} n+p$ involving nucleons, apparently, in this type of reaction the quark structure of the nucleons is not affected, so the probability of occurring the metastable nucleus ${ }^{2} n_{\text {isu }}$ is extremely small. Therefore, estimates of the smallness of the contribution of the dineutron to this process, made in $[5,6]$, should be attributed to the possible contribution of the $\beta$-dineutron.

\subsection{Mechanism of $\mathrm{e}^{-}$-catalysis}

Another way of initiating the $\alpha$-decay of tungsten isotopes under the conditions of glow discharge is 
also possible (see above) during $\mathrm{e}^{-}$-catalysis, when electrons with kinetic energy $E_{\mathrm{e}} \sim 3-5 \mathrm{eV}$ interact directly with stable isotopes of tungsten. Possible examples of such processes are given below:

$$
\begin{aligned}
& { }_{74}^{183} \mathrm{~W}+e^{-} \rightarrow{ }_{73}^{183} \mathrm{Ta}_{i s u}+v \rightarrow \\
& \rightarrow{ }_{71}^{175} \mathrm{Lu}+2{ }_{2}^{4} \mathrm{He}+2 e^{-}+2 \tilde{v}+v+Q(3.96 \mathrm{MeV}), \\
& { }_{74}^{186} \mathrm{~W}+e^{-} \rightarrow{ }_{73}^{186} \mathrm{Ta}_{i s u}+v \rightarrow \\
& \rightarrow{ }_{70}^{174} \mathrm{Lu}+3{ }_{2}^{4} \mathrm{He}+3 e^{-}+3 \tilde{v}+v+Q(7.17 \mathrm{MeV}) .
\end{aligned}
$$

It should be pointed out that, using the concepts of $\mathrm{e}^{-}$-catalysis, it can be understood that not all the new isotope products detected in the experiment are obtained. Therefore, processes involving the ${ }^{2} n_{\text {isu }}$ nuclei are considered for the initiation of the $W$ stable isotope decays as the basic ones.

The above data allow us to conclude that the nuclear decay of initially non-radioactive tungsten isotopes with the formation of lighter elements (erbium, lutetium, ytterbium, hafnium), initiated under the condition of low-temperature plasma (glow discharge), can be considered as a new type of initiated decay of nuclei that does not result from nuclear reactions, for example, with alpha particles or nucleons, but under the action of lowenergy electrons. It should be remembered that stable isotopes of many nuclei, from neodymium to bismuth and including the tantalum-181 isotope in particular, for which initiated decay processes similar to the ones described were also observed in $[8,9]$, are potentially $\alpha$-radioactive in the same sense as tungsten isotopes.

\subsection{HARPOON MECHANISM}

The extreme difficulties in understanding the mechanism of low-energy nuclear processes are the reactions between many-electron atoms. Such processes are usually considered in connection with the study of transformation processes in native systems [31-33]. However, it was recently shown [34] that reactions of this type can occur during the initiation of self-propagating hightemperature synthesis (SHS) processes [35]. The composition of condensed combustion products of thermite powder mixtures $\left(\mathrm{Al}+\mathrm{Fe}_{2} \mathrm{O}_{3}\right)$ in air was investigated in [34]. The purity of the starting materials was (99.7-99.9)\% mass. It was shown that in the process of termite combustion (flame temperature exceeded $2800 \mathrm{~K}$ ) calcium is formed and stabilized in an amount up to $0.55 \%$ mass. In the initial thermite powder systems $\left(\mathrm{Al}+\mathrm{Fe}_{2} \mathrm{O}_{3}\right)$, calcium was absent. According to [34], calcium could be formed in nuclear reactions

$$
\begin{aligned}
& { }_{13}^{27} \mathrm{Al}+{ }_{7}^{14} \mathrm{~N} \rightarrow{ }_{20}^{41} \mathrm{Ca}+Q(21.8 \mathrm{MeV}), \\
& { }_{13}^{27} \mathrm{Al}+{ }_{7}^{14} \mathrm{~N} \rightarrow{ }_{20}^{40} \mathrm{Ca}+n+Q(12.44 \mathrm{MeV}) .
\end{aligned}
$$

The phenomenon of calcium formation in experiments [34] may indicate that in the flame of iron-aluminum thermite combustion in air, the electron temperature can be much higher than the flame temperature, fixed by the energy of atoms and ions. It is the latter situation that is typical of a low-temperature glow discharge plasma. In this case, the interaction of high-energy electrons with nuclei ${ }_{13}^{27} \mathrm{Al}$ and ${ }_{7}^{14} \mathrm{~N}$ could lead to the formation of nuclei ${ }_{13}^{27} M g_{i s u}$ and ${ }_{6}^{14} C_{i s u}$, accordingly. The largest activity in nuclear interactions from these nuclei is inherent to the nucleus ${ }_{13}^{27} M g_{\text {isu }}$, since the deficit of its energy relative to the nucleus ${ }_{13}^{27} \mathrm{Mg}$ is $\Delta Q=-2.61 \mathrm{MeV}$, whereas for the nucleus ${ }_{6}^{14} C_{i s u}$ the corresponding value is much less and equal to $-0.16 \mathrm{MeV}$.

We will assume, following [20], that if the nucleus of an atom (or ion) is in a metastable, predecay isu-state (we assume that this is ${ }_{13}^{27} M g_{i s u}$ ), then the lability of the electronic subsystem increases, and the probability of its partial overlap with electronic subsystems of the located near atom (in this case, the nitrogen atom), grows for this atom. It is obvious that large values of the energy releases of the gross processes (22) and (23) should act as an initiating factor to the manifestation of the spin-spin interaction of the electronic subsystems of both atoms and the formation of common "molecular" orbitals under the correcting action of spin electronnuclear interactions for each of the atoms. The emerging bonds pull both atoms together, and the formation of common orbitals is more intense as the nuclei are brought closer together. As a result, a sort of "harpoon mechanism" is observed when a neighboring atom is captured by the atom with the nucleus in the isu-state. The complete integration of the electronic subsystems of both atoms initiates the fusion of the nuclear matter of 
the nucleus in the isu-state (in our case ${ }_{13}^{27} M g_{\text {isu }}$ ) and the adjacent nucleus (in our case ${ }_{7}^{14} \mathrm{~N}$ ). The corresponding brutto-process can be presented as:

$$
\begin{aligned}
& { }_{13}^{27} \mathrm{Al}+{ }_{7}^{14} \mathrm{~N}+e_{b e}^{-} \rightarrow \\
& \rightarrow{ }_{20}^{41} \mathrm{Ca}+e^{-}+v+\tilde{v}+Q(21.8 \mathrm{MeV}), \\
& { }_{13}^{27} \mathrm{Al}+{ }_{7}^{14} \mathrm{~N}+e_{b e}^{-} \rightarrow \\
& \rightarrow{ }_{20}^{40} \mathrm{Ca}+n+e^{-}+v+\tilde{v}+Q(12.44 \mathrm{MeV}) .
\end{aligned}
$$

Earlier, the harpoon mechanism was considered in connection with the processes of nuclear transmutations in native systems [20].

Because of the manifestation of weak nuclear interactions in the formation of the nuclear matter of the final nucleus as a set of interacting nucleons, a significant part of the energy release can also be realized by emitting neutrinos and antineutrinos if the final nucleus can be formed in the ground state by virtue of spin and parity conservation laws. Of course, in cases where the final nuclei are formed in an excited state, along with non-ionizing radiation of neutrinos and antineutrinos, X-rays or gamma quanta will be emitted. Under the experimental conditions [34], $\mathrm{X}$-rays were detected.

Our phenomenological analysis shows that in order to understand the essence of the observed nuclear transformations during burning of thermite mixtures, it is actual to develop new theoretical approaches not to the calculation of the quantum mechanical probabilities of certain processes, but rather to modeling the dynamics of nuclear processes on the basis of quantum-chemical analysis. By this we mean calculations of the electronic structure of an atom upon the formation of nuclei in the isu-state with disturbed nucleon structure; calculations for modeling the spatial instability of the electronic subsystem of an atom that emerges due to the loss of nucleus stability; and calculations for the dynamics of the overlapping of such mobile orbitals with the electron orbitals of neighboring atoms and the formation of molecular orbitals that initiate the convergence and fusion of the respective nuclei. Kramer's activation mechanism in its discrete version (the random walk on the energy levels of the system to reach a set boundary) [36] generally used in physicochemical kinetics could be useful in analyzing the dynamics of nuclear radioactive decay. Here we mean the dynamics of the accumulation of energy by the nucleus in the unstable isu-state on the "last" bond, the disruption of which means the decay of the nucleus along a certain channel.

\section{NUCLEAR CHEMICAL PROCESSES IN ANDREA ROSSI'S E-CAT REACTOR}

Within the framework of the developed ideas on the mechanisms for initiating low-energy nuclear reactions, the results obtained in the testing of A. Rossi's energy E-Cat installations can also be understood [21]. Here we briefly discuss the test results of the E-Cat working element of the A. Rossi reactor, presented by a group of international experts [22]. The working element was a hollow ceramic tube $2 \mathrm{~cm}$ in diameter and $20 \mathrm{~cm}$ long, into which fuel was loaded - about $0.9 \mathrm{~g}$ of finely dispersed nickel (all stable isotopes were present, $-{ }_{28}^{58} \mathrm{Ni},{ }_{28}^{60} \mathrm{Ni},{ }_{28}^{61} \mathrm{Ni},{ }_{28}^{62} \mathrm{Ni}$ and ${ }_{28}^{64} \mathrm{Ni}$, whose abundances were $67,26.3,1.9,3.9$ and $1 \%$, respectively), and $0.1 \mathrm{~g}$ of $\mathrm{LiAlH}_{4}$ powder ( ${ }_{3}^{6} \mathrm{Li}$ and ${ }_{3}^{7} \mathrm{Li}$ isotopes, whose abundances were 8.6 and $91.4 \%$, respectively). The tube was sealed and then heated. The tests were carried out for 32 days at element heating temperatures up to $1260^{\circ} \mathrm{C}$ (first half of the time) and $1400^{\circ} \mathrm{C}$ (second half of the time). The energy released during the tests was measured from the value of the heat flux emanating from the element. During the test, excess energy was 1.5 MWh, so the efficiency exceeded 3.5. Changes were recorded in the isotopic composition of the main components of the "fuel" - nickel and lithium, the initial abundances of the stable elements of which was close to the tabulated natural abundances. After the tests, the isotopic composition of the fixed elements has changed greatly: almost all of the nickel (more than 98\%) was represented by the nickel-62 isotope (about $4 \%$ in the initial abundances), and the fraction of lithium- 7 decreased to almost $8 \%$, so that the fraction of the lithium- 6 practically increased 
Table 1 fuel elements (aluminum and nickel isotopes) with

The isotope abundances of the initial "fuel" and "ash" after the tests [22]

\begin{tabular}{|c|c|c|c|c|c|}
\hline \multirow[t]{2}{*}{ Ion } & \multicolumn{2}{|c|}{ Fuel } & \multicolumn{2}{|c|}{ Ash } & \multirow{2}{*}{$\begin{array}{c}\text { Natural } \\
\text { abundance } \\
{[\%]}\end{array}$} \\
\hline & $\begin{array}{l}\text { Counts } \\
\text { in peak }\end{array}$ & $\begin{array}{c}\text { Measured } \\
\text { abundance } \\
{[\%]}\end{array}$ & $\begin{array}{l}\text { Counts } \\
\text { in peak }\end{array}$ & $\begin{array}{c}\text { Measured } \\
\text { abundance } \\
{[\%]}\end{array}$ & \\
\hline${ }^{6} \mathrm{Li}^{+}$ & 15804 & 8.6 & 569302 & 92.1 & 7.5 \\
\hline${ }^{7} \mathrm{Li}^{+}$ & 168919 & 91.4 & 48687 & 7.9 & 92.5 \\
\hline${ }^{58} \mathrm{Ni}^{+}$ & 93392 & 67 & 1128 & 0.8 & 68.1 \\
\hline${ }^{60} \mathrm{Ni}^{+}$ & 36690 & 26.3 & 635 & 0.5 & 26.2 \\
\hline${ }^{61} \mathrm{Ni}^{+}$ & 2606 & 1.9 & $\sim 0$ & 0 & 1.8 \\
\hline${ }^{62} \mathrm{Ni}^{+}$ & 5379 & 3.9 & 133272 & 98.7 & 3.6 \\
\hline${ }^{64} \mathrm{Ni}^{+}$ & 1331 & 1 & $\sim 0$ & 0 & 0.9 \\
\hline
\end{tabular}

to $92 \%$. Data on the isotope abundances of the initial "fuel" and "ash" after the tests are given in Table 1 [22].

In accordance with the developed concepts, a fixed change in the isotopic composition of the base elements of the "fuel" - nickel and lithium in the presence of hydrogen liberated during the decomposition of $\mathrm{LiAlH}_{4}$ at given temperatures may be due to the formation of protium-containing plasma in the reaction space and the formation of neutral metastable nuclei ${ }^{1} n_{\text {isu }}$. We believe that the half-life of such nuclei, as well as of nuclei ${ }^{2} n_{\text {isu }}$, is sufficiently large, so that such neutral nuclei, like neutrons, can interact with nuclei of elements of the elements that make up the fuel, determining the occurring changes in the elemental and isotopic composition of the fuel, which are accompanied by the corresponding energy release:

$$
\begin{aligned}
& { }_{3}^{7} \mathrm{Li}+{ }^{1} n_{i s u} \rightarrow 2{ }_{2}^{4} \mathrm{He}+e^{-}+\tilde{v}+v \tilde{v}+Q(17.35 \mathrm{MeV}) \\
& { }_{13}^{27} \mathrm{Al}+{ }^{1} n_{i s u} \rightarrow{ }_{2}^{4} \mathrm{He}+{ }_{12}^{24} \mathrm{Mg}+e^{-}+\tilde{v}+v \tilde{v}+Q(1.60 \mathrm{MeV}) \\
& { }_{13}^{27} \mathrm{Al}+{ }^{1} n_{i s u} \rightarrow{ }_{14}^{28} \mathrm{Si}+e^{-}+\tilde{v}+v \tilde{v}+Q(11.58 \mathrm{MeV}), \\
& { }_{28}^{58} \mathrm{Ni}+{ }^{1} n_{i s u} \rightarrow{ }_{2}^{4} \mathrm{He}+{ }_{25}^{55} \mathrm{Mn}+e^{+}+v+v \tilde{v}+Q(2.35 \mathrm{MeV}), \\
& { }_{28}^{60} \mathrm{Ni}+{ }^{1} n_{i s u} \rightarrow{ }_{2}^{4} \mathrm{He}+{ }_{26}^{57} \mathrm{Fe}+v \tilde{v}+Q(0.47 \mathrm{MeV}), \\
& { }_{28}^{61} \mathrm{Ni}+{ }^{1} n_{i s u} \rightarrow{ }_{2}^{4} \mathrm{He}+{ }_{26}^{58} \mathrm{Fe}+v \tilde{v}+Q(2.80 \mathrm{MeV}), \\
& { }_{28}^{62} \mathrm{Ni}+{ }^{1} n_{i s u} \rightarrow{ }_{2}^{4} \mathrm{He}+{ }_{27}^{59} \mathrm{Co}+e+\tilde{v}+v \tilde{v}+Q(0.34 \mathrm{MeV}), \\
& { }_{28}^{64} \mathrm{Ni}+{ }^{1} n_{i s u} \rightarrow{ }_{29}^{65} \mathrm{Cu}+e^{-}+\tilde{v}+v \tilde{v}+Q(7.45 \mathrm{MeV}) .
\end{aligned}
$$

As follows from this list of reactions, the largest specific (per unit mass of the component) energy release is associated with the nuclei of lithium-7. However, the total contribution to heat release during nuclear reactions ${ }^{1} n_{\text {isu }}$ with all other a small mass fraction of the lithium-7 isotope in the system can be the main one. The fixed almost complete disappearance of isotopes ${ }_{3}^{7} \mathrm{Li}$ and ${ }_{28}^{58} \mathrm{Ni}$ in the ashes after monthly tests of the module indicates rather high rate constants of not only processes (24) and (27), but also of the other nuclear processes that take place with the formation of new chemical elements.

In order to understand the specific causes of the main fuel composition changes during the operation of the E-Cat unit, which consist in almost complete exhaustion of the lithium-7 isotope, and also in the overwhelming increase the nickel-62 isotope in the ash, it is necessary to indicate other nuclear reactions that also led to a change in the isotopic composition of the initial nickel. These reactions were accompanied by a significantly lower heat release than the above reactions, due to energy losses carried away by the neutrinos and antineutrinos formed:

$$
\begin{aligned}
& { }_{28}^{58} \mathrm{Ni}+{ }^{1} n_{i s u} \rightarrow{ }_{28}^{59} \mathrm{Ni}+v \tilde{v}+Q(8.22 \mathrm{MeV}), \\
& T_{1 / 2}\left({ }_{28}^{59} \mathrm{Ni}\right)=7.6 \times 10^{4} \mathrm{yr}, \\
& { }_{28}^{60} \mathrm{Ni}+{ }^{1} n_{i s u} \rightarrow{ }_{28}^{61} \mathrm{Ni}+v \tilde{v}+Q(7.04 \mathrm{MeV}), \\
& { }_{28}^{61} \mathrm{Ni}+{ }^{1} n_{i s u} \rightarrow{ }_{28}^{62} \mathrm{Ni}+v \tilde{v}+Q(9.81 \mathrm{MeV}), \\
& { }_{28}^{62} \mathrm{Ni}+{ }^{1} n_{i s u} \rightarrow{ }_{28}^{63} \mathrm{Ni}+v \tilde{v}+Q(6.05 \mathrm{MeV}), \\
& T_{1 / 2}\left({ }_{28}^{63} \mathrm{Ni}\right)=100.1 \mathrm{yr}, \\
& { }_{28}^{64} \mathrm{Ni}+{ }^{1} n_{i s u} \rightarrow{ }_{28}^{65} \mathrm{Ni}+v \tilde{v}+Q(5.32 \mathrm{MeV}), \\
& T_{1 / 2}\left({ }_{28}^{65} \mathrm{Ni}\right)=2.52 \mathrm{~h} .
\end{aligned}
$$

First of all, let us point out the large value of the half-life of the isotope ${ }_{28}^{59} \mathrm{Ni}$, which practically excludes the "charging" of other decaying isotopes of nickel during the tests due to the isotope ${ }_{28}^{58} \mathrm{Ni}$, whose fraction is 2 times that of the other isotopes of nickel. For this reason, the almost complete absence of the isotope ${ }_{28}^{60} \mathrm{Ni}$ in the sol should be associated with the process (33) only, because of the relatively low energy release in the process (28). It can also be assumed that the processes (29) and (34) cause the disappearance of the isotope ${ }_{28}^{61} \mathrm{Ni}$ in the ash, but the process (34) with high energy release thus feeds the ash with an isotope ${ }_{28}^{62} \mathrm{Ni}$, causing the the prevailing abundance of this 
isotope among the other isotopes of nickel in the ash. The last factor is the "small" rate constant for the disappearance of the isotope ${ }_{28}^{62} \mathrm{Ni}$ due to reaction (30) of cobalt formation with small energy release in this process. It is also important to point out that the long half-life of the isotope ${ }_{28}^{63} \mathrm{Ni}$ practically excludes the completion of the ash with an isotope ${ }_{28}^{64} \mathrm{Ni}$ and process (31) with high energy release provides a practically complete transition of this isotope in the initial nickel to the copper-65 isotope.

Of course, here only qualitative arguments are given in favor of the fixed ash composition after the test. The comparative study of the elemental and isotopic composition of the initial fuel and ash by using mass spectrometry with inductively coupled plasma technique [37], previously effectively used in the investigation of the isotopic composition of impurities in nickel upon laser ablation of a nickel sample in an aqueous medium, could become more relevant. Here, it is important to study the changes in the isotopic ratios for different elements in the initial fuel and in the ash, primarily for the base element of nickel, as well as the elements formed in accordance with the processes (24)-(31)first of all, silicon-28 and copper-65.

\section{CONCLUDING REMARKS}

It is known that the results of [1] were not accepted by many physicists, not only because of difficulties in understanding the mechanism of formation of neutrons and tritium in the electrolysis of heavy water $\mathrm{D}_{2} \mathrm{O}$ with $\mathrm{Pd}$-cathode, but also because of the irreproducibility of the results [1] in various laboratories. In what can the reason, undoubtedly objective, be so irreproducible? It seems to the author that the main difficulty in reproducing the results of [1] is to create conditions under which electrons injected from the surface of the Pd-cathode participating in nuclear chemical processes must reach high (by chemical scales) kinetic energies $E_{\mathrm{e}} \sim 3-5 \mathrm{eV}$. It can be assumed [16,38] that under the conditions of reproducible experiments [7-13], these energies are achieved due to the presence of the spike-like random irregularities of the surface nanorelief on the metal surface that change sharply in height "roughness". The such nanoheterogeneity of the metal surface, namely, the cathode in a glow discharge in a deuteriumcontaining gas medium of ions and molecules, and the surface of a metallic sample during laser ablation in aqueous media [10-13] is the result of impacts of plasma ions and molecules on the surface of metallic samples.

In the latter cases, the roughness of the metal surface is sufficient to initiate nuclear transformations even in the absence of cathodic displacement. At cathodic displacement, the efficiency of nuclear chemical processes increases by orders of magnitude, as was shown in [13]. We indicate factors that cause an increase in the flux of electron emission from the surface of metals and an increase in the kinetic energy of electrons emerging from the metal in the vicinity of the spike-like random irregularities on the metal surface. First of all, increased values of mechanical tensile stresses are realized in these regions, which leads to a decrease in the work function of the electron from the metal (the Zhurkov effect [39]) and causes an increase in the flux of electrons from metals. In addition to this, electric fields of the highest intensity, which not only lower the energy barrier for the injection of electrons from the metal (the Frenkel effect [40]), but also cause the acceleration of the emitted electrons, are localized in the vicinity of the most abrupt changes in the surface profile - in the vicinity of the spike-like random irregularities. Here we also point to the paper [26], in which a decrease in the effective work function of electrons from the surface of tungsten cathodes was observed for thermionic emission initiated by laser radiation of pico- and femtosecond durations.

The conclusions about the interrelation between the efficiency of nuclear transformations observed in [10-13] and the features of the metal surface structure in the nanometer range are directly confirmed by the results of [38]. In this work, the method of atomic force microscopy was used to analyze the surface relief of metals formed under different regimes of laser ablation in an aqueous medium. It has been shown that 
nuclear transformations occur only under intensive impacts of plasma ions and molecules on the surface, when the "roughness" greatly increases and the texture "spikiness factor" as one of the key $3 D$ parameters of the surface relief in the nanometer range $[42,43]$ determining the measure of the most abrupt changes of the surface profile becomes significant.

If under the conditions of laser ablation of metals in aqueous media and in a glow discharge, the level of nanoheterogeneity of the surface necessary for the injection of highenergy electrons into the medium is maintained by the impact of ion and atom fluxes on metal surfaces, under conditions of electrolysis of heavy water with a Pd cathode there is no such factor. Probably, when carrying out appropriate studies of the surface relief and the structure of fractured near-surface layers of $\mathrm{Pd}$-cathodes under conditions of $\mathrm{D}_{2} \mathrm{O}$ electrolysis, it will be possible to find the regimes of the electrolysis process when the reproducibility of appearance of the neutrons and tritium nuclei in this process is reached.

\section{REFERENCES}

1. Fleishmann $\mathrm{M}$, Pons $\mathrm{S}$, Hawkins $\mathrm{M}$. Electrochemically induced nuclear fusion of deuterium. J. Electroanal. Chem., 1989, 261:301-308.

2. Timashev SF. On possible mechanism of nuclear-chemical transformation in $\mathrm{Pd}$ electrode under electrolysis in heavy water. Journal of Physical Chemistry, 1989, 63(8):22832284 (in Russ.).

3. Sakisaka M, Tomita M. Experiments on the possible existence of a bound di-neutron. J. Phys. Soc. Japan, 1961, 16:2597-2598.

4. Katase A, Seki M, Akiyoshi, Yoshimura A, Sonoda M. Experiments on the existence of dineutrons. J. Phys. Soc. Japan, 1962, 17:1211-1212.

5. Gol'danskii VI, Zel'dovich YaB, Baz' AI. Systematics of the lightest nuclei. Phys.Usp. Advances in Physical Sciences, 1965, 8(2):177-201.

6. Borzakov SB, Panteleev TS, Strelkov AV, Soldner T. The Search for the Dineutron at the
High Flux Reactor ILL (Grenoble, France). ISINN-10, JINR, 2003.

7. Savvatimova I. Transmutation of elements in low-energy glow discharge and the associatedprocesses. Condensed Matter Nucl. Sci., 2011, 8:1-19.

8. Savvatimova I. Creation of more light elements in tungsten irradiated by low-energy deuterium ions. Proc. 13th Int. Conf. ICCF13, Sochy, Russia, 2007:505-517.

9. Savvatimova I, Savvatimov G, Kornilova A. Decay in tungsten irradiated by low energy deuterium ions. Proc. 13th Int. Conf. ICCF13, Sochy, Russia, 2007:295-308.

10. Shafeev GA, Bozon-Verduraz F, Robert M. Experimental evidence of transmutation of $\mathrm{Hg}$ into $\mathrm{Au}$ under laser exposure of $\mathrm{Hg}$ nanodrops in $\mathrm{D}_{2} \mathrm{O}$. Physics of Wave Phenomena, 2007, 15(3):131-136.

11. Simakin AV, Shafeev GA. Initiation of nuclear reactions under laser irradiation of metal nanoparticles in the presence of thorium aqua ions. Physics of Wave Phenomena, 2008, 16(4):268-274.

12. Barmina EV, Sukhov IA, Lepekhin NM, Priseko YuS, Filippov VG, Simakin AV, Shafeev GA. Application of copper vapor lasers in control of activity of Uranium isotopes. Quantum Electronics, 2013, 43:591-596.

13. Barmina EV, Timashev SF, Shafeev GA. Laserinduced synthesis and decay of Tritium under exposure of solid targets in heavy water. Journal of Physics: Conference Series, 2016, 688:012106. (8th Intern. Conf. on Inertial Fusion Sciences and Applications (IFSA 2013) IOP Publishing; /http://arxiv.org/abs/1306.0830 [physics. gen-ph]).

14. Seth KK, Parker B. Evidence for dineutrons in extremely neutron-rich nuclei. Phys. Rev. Lett., 1991, 66(19):2448-2451.

15. Akhiezer AI, Rekalo MP. Elementarnye chastitsy [Elementary particles]. Moscow, Nauka Publ., 1986, 256 p.

16. Timashev SF. Physical vacuum as a system manifesting itself on various scales - from nuclear physics to cosmology; http://arxiv. org/abs/1107.1799v7. 
17. Timashev SF, Simakin AV, Shafeev GA. Nuclear-Chemical processes under the conditions of laser ablation of metals in aqueous media (Problems of "cold fusion"). Russian Journal of Physical Chemistry A., 2014, 88(11):1980-1988).

18. Timashev SF. Radioactive decay as a forced nuclear chemical process: phenomenology. Russian Journal of Physical Chemistry A., 2015, 89(11):2072-2083.

19. Timashev Serge. Nuclear-chemical processes in the solar atmosphere. International Journal of Astrophysics and Space Science, 2014, 2(6):88-92.

20. Timashev SF. Initiating nuclear-chemical transformations in native systems: phenomenology. Russian Journal of Physical Chemistry A, 2016, 90(10):2089-2095.

21. United State Patent - US 9,115,913 B1, Aug. 25. 2015; https://animpossibleinvention.files. wordpress.com/2015/08/us9115913b1.pdf; Andrea Rossi.

22. Levi G, Foschi E, Höistad B, et al. Observation of Abundant Heat Production from a Reactor Device and of Isotopic Changes in the Fuel. http://amsacta.unibo.it/4084/1/ LuganoReportSubmit.pdf.

23. Jung M, Bosch F, Beckert K, et al. First observation of bound-state decay. Phys. Rev. Lett., 1992, 69:2164-2167.

24. Bosch F, Faestermann T, Friese J, et al. Observation of bound-state $\beta^{-}$-decay of fully ionized ${ }^{187} \mathrm{Re}:{ }^{187} \mathrm{Re}-{ }^{187}$ Os cosmochronometry. Phys. Rev. Lett., 1996, 77:5190-5193.

25. Thomas SA, Abdalla FD, Lahav O. Upper Bound of $0.28 \mathrm{eV}$ on Neutrino Masses from the Largest Photometric Redshift Survey. Phys. Rev. Lett., 2010, 105(3):031301.

26. Lattimer JM, Pethick CJ, Prakash M, Haensel P. Direct URCA process in neutron stars. Phys. Rev. Lett., 1991, 66:2701-2704.

27. Timashev Serge F. The Planck numbers and the essence of gravity: phenomenology. http:// arxiv.org/abs/1701.08073 [physics.gen-ph].

28. Vysotskii VI, Kornilova AA. Microbial transmutation of Cs-137 and LENR in growing biological systems. Curr. Sci., 2015, 108:636-640.
29. Donnelly TW, Formaggio JA, Holstein BR, Milner RG, Surrow B. Foundations of Nuclear and Particle Physics. Cambridge, University Press, 2017, 745 p.

30. Sharapov VM, Kanashenko SL. Vopr. At. Nauki Tekh., Ser. Termoyad. Sintez. 2008, 2:20 (in Russ.).

31. Kervran CL. Biological Transmutation. Happiness Press, USA, Magalia, California,1998.

32. Biberian J-P. Review Article Biological Transmutations: Historical Perspective. J. Condensed Matter Nucl. Sci., 2012, 7:11-25.

33. Vysotskii VI, Kornilova AA. Nuclear Transmutation of Stable And Radioactive Isotopes In Biological Systems. Pentagon Press, New Delhi, 2010.

34. Gromov AA, Gromov AM, Popenko EM, et al. Formation of calcium in the products of iron-aluminum thermite combustion in air. Russian Journal of Physical Chemistry A, 2016, 90(10):2104-2106.

35. Borisov AA, De Luca LT, Merzhanov AG. Self-Propagating High-Temperature Synthesis of Materials. CRC Press, Taylor and Francis, 2000, $400 \mathrm{p}$.

36. Tunitskii NN, Kaminskii VA, Timashev SF. Metody fisikokbimicheskoy kinetiki [Methods of Physicochemical Kinetics]. Moscow, Khimiya Publ., 1972, 198 p.

37. Revel'skiy IA, Buryak AK, Sajti PL, et al. Isotopic ratio changes of several trace elements in nickel as a result of laser ablation in aqueous medium, in press.

38. Serkov AA, Akovantseva AA, Barmina EV. Influence of the Surface Structure on the Initiation of Nuclear Chemical Processes Under Laser Ablation of Metals in Aqueous Media. Russian Journal of Physical Chemistry A, 2014, 88(11):1989-1995.

39. Timashev SF. On the thermofluctuation nature of the strength of solids. Reports of the USSR Academy Science, 1984, 276:898-902 (in Russ.).

40. Bonch-Bruevich VL, Kalashnikov SG. Fizika poluprovodnikov [Physics of Semiconductors]. Moscow, Nauka Publ., 1990, 678 p.

41. Barmina EV, Fotakis C, Stratakis E, Stolyarov VN, Stolyarov IN, Shafeev GA, Serkov AA. 
Nano-textured W shows improvement of thermionic emission properties. Appl. Phys. A, 2012:1-4.

42. Timashev SF, Polyakov YuS, Lakeev SG, Misurkin PI, Danilov AI. Fundamentals of fluctuation metrology. Russian Journal Physical Chemistry A., 2010, 84(10):1807-1825.

43. Mirsaidov U, Timashev SF, Polyakov YuS, Misurkin PI, Polyakov SV, Musaev I. Analytical method for parameterizing the random profile components of nanosurfaces imaged by atomic force microscopy. Analyst, 2011, 136(3):570576; http://arxiv.org/abs/1010.6232. 